\title{
Optical diffraction of focused spots and subwavelength structures
}

\author{
D. S. Marx* and D. Psaltis \\ California Institute of Technology, Department of Electrical Engineering, Mail Code 136-93, \\ Pasadena, California 91125
}

Received August 9, 1996; revised manuscript received December 20, 1996; accepted January 2, 1997

\begin{abstract}
We have developed a numerical diffraction tool for cases in which the incident field is a focused spot and the diffracting structure is a single structure or an aperiodic surface. Our approach uses the integral formulation to solve Maxwell's equations and is different from previously published methods in its choice of basis function. We compared numerical results with experimental measurements of the far-field intensity for a focused spot incident on an aluminum grating, and the comparison was favorable. Finally, we predict the diffraction behavior of the proposed digital video disk format for the next generation of optical disk. Our analysis shows that the reflected signal for this format has a strong dependence on the polarization of the incident light. (C) 1997 Optical Society of America [S0740-3232(97)02706-3]

Key words: vector diffraction analysis, optical disk (disk) memories.
\end{abstract}

\section{INTRODUCTION}

The numerical calculation of rigorous electromagnetic diffraction is a broad field with many different approaches and applications. In general, a particular approach must be carefully selected and tailored to the specific problem at hand. Our desire in the present effort is to study the diffracted far field that results when a high-numericalaperture, diffraction-limited, and focused spot is incident on a single or aperiodic structure. Examples of diffraction problems that meet this description are optical memories and critical dimension metrology in microelectronics fabrication. Previous analysis of optical disk memories and other random or rough surfaces has typically described the incident field as a plane wave. ${ }^{1-6}$ As the numerical aperture of the incident light increases, as is the case for the new digital video disk (DVD) format, this approximation becomes less satisfactory.

Feature sizes in microelectronics fabrication are now approaching $0.25 \mu \mathrm{m}$, and they are expected to be as small as $0.18 \mu \mathrm{m}$ by $2000 .^{7}$ While current efforts exist in the development of scanning electron microscopes and atomic force microscopes for critical dimension measurement in a mass production environment, optics will always have the advantage in speed and cost. However, the image resolution limit for optical instruments is very severe. Efforts to measure optically characteristics of unresolvable features include scatterometry. ${ }^{8,9}$ The theoretical approach in this method considers the problem of a plane wave incident on a grating. Our contribution is an attempt to model the measurement of a single or aperiodic structure with a focused spot. The advantage of this approach over scatterometry is that single features or lines can be measured, rather than parameters of a grating.

The basic principle behind the use of optical systems to measure features that are normally unresolvable is the use of a priori information. For example, an optical memory format is known when the read head is designed, and, in fact, the optical memory format is purposely designed to maximize the read signal. However, when an astronomical observation of a star is made, for example, nothing is known about the star except what can be observed. Therefore the astronomer is limited to the classical resolution, while an optical disk player can make measurements of pits that are half the wavelength in diameter. In the middle of these two extreme examples, in terms of a priori information, is the problem of critical dimension measurements of microelectronics circuits. In this case the intended pattern is known beforehand, but it is designed for its electrical operation, not for its optical properties.

Our diffraction model (Fig. 1) consists of a substrate surface with a known contour and an incident field resulting from a beam focused through an objective with a known numerical aperture. All the results presented here are for two-dimensional structures. When the geometry of the structure is two dimensional, the problem decomposes into two independent polarizations. We define the TE polarization as the case in which the electric field is perpendicular to the plane of incidence and parallel to the groove or the line, and the TM polarization is for the electric field lying in the plane of incidence.

There are several numerical vector diffraction methods described in various publications. We want to select the method that best suits our particular problem. Most numerical methods are designed for periodic structures and plane-wave incidence. For example, the integral method invented by Petit and improved on by Wirgin, ${ }^{10-13}$ the coupled-mode methods, ${ }^{14,15}$ and the coupled-wave methods ${ }^{16-19}$ make these assumptions. To use a method that requires periodicity, we could approximate a single structure with a periodic structure with a very long period. The diffraction for a focused spot would then be calculated by considering separately each plane wave that composes the focused incident field. However, the number of orders required in the diffraction calculation, be- 


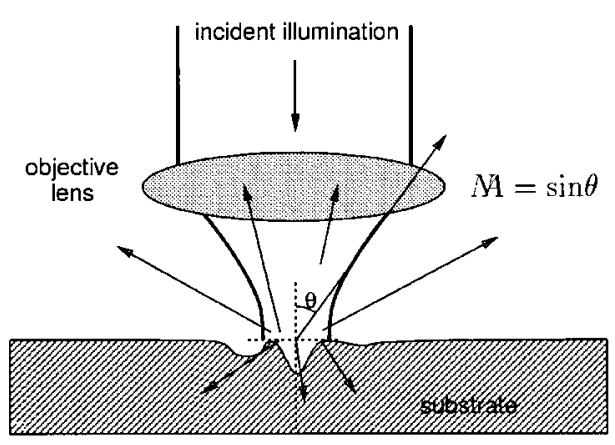

Fig. 1. Basic diffraction model addressed in this paper. NA stands for numerical aperture.

cause of the large period, will cause a burden on time and memory of the computation, and this computation time must be multiplied by the number of plane waves that are to be considered in composing the focused spot. Therefore we decided to pursue a more direct integration technique that will allow us to calculate the diffraction directly from the focused incident field and a single groove.

The two most general approaches to numerical solutions of Maxwell's equations are the integral approach and the differential approach. Examples of some differential approaches are finite differences, finite-element analysis, and the more recent finite-difference time domain method. In general, these methods are best when the problem is contained within finite boundaries or the medium is inhomogeneous. For the particular problem addressed here, the diffracting structure is typically an aluminum contour covered with polycarbonate (an optical disk) or a structure etched into silicon or its dielectric film. The most natural approach to calculate diffraction for a structure that can be described as a contour seems to be the integral method as described by de Hoop. ${ }^{20}$

Since the integral method for numerical diffraction calculations is well described in the literature, ${ }^{1,2,10-13,20}$ we will limit our discussion of the formulation of the numerical method to those details in which our method differs from that of the references. Our adaptations all relate to our specific diffraction problem of a focused spot and a single, nonperiodic structure. In the next section, we will the basis function and checks for aliasing resulting from inadequate grid spacing. Section 3 is devoted to the comparison of numerical result with experimental measurements. For our comparison we use a focused spot formed by a high-numerical-aperture objective incident on an aluminum grating. The incident light is polarized so that the electric field is parallel to the grating vector (perpendicular to the groove wall), so that the Wood's anomaly is excited. In Section 4 we make a prediction about the polarization properties of the new DVD format. The numerical results for the format lead to a proposal for the doubling of storage density on the disk.

\section{NUMERICAL SOLUTION}

\section{A. Coupled Integral Equations}

The formulation for the integral method of numerical diffraction is described in detail by de Hoop ${ }^{20}$ and Bouwhuis et $a l .{ }^{2}$ and specific operator matrices for the two polarizations in two-dimensional diffraction problems are given in the appendix of Dil and Jacobs. ${ }^{1}$ For the purpose of describing the differences between our numerical method and these references, we will start with the coupled integral equations derived from Maxwell's equations in the references. The unknown quantities in the integral equations are the tangential electric and magnetic fields at the boundary between two media. The boundary is described by a surface in three dimensions and a contour in two dimensions. The tangential electric and magnetic fields can be defined as the magnetic and electric surface currents

$$
\mathbf{J}\left(\mathbf{r}_{s}\right)=\left(\begin{array}{c}
\mathbf{J}_{s, m}\left(\mathbf{r}_{s}\right) \\
\mathbf{J}_{s, e}\left(\mathbf{r}_{s}\right)
\end{array}\right)=\left(\begin{array}{c}
\hat{n} \times \mathbf{E}\left(\mathbf{r}_{s}\right) \\
-\hat{n} \times \mathbf{H}\left(\mathbf{r}_{s}\right)
\end{array}\right),
$$

where $\mathbf{r}_{s}$ is a point on the surface and $\hat{n}$ is a unit vector normal to the surface and pointing into the incident medium. The fields in either medium and on the surface are expressed as superposition integrals of the surface currents on the surface and the Green's function. In operator notation the tangential fields at a field point $\mathbf{r}$ are

$$
\mathbf{J}(\mathbf{r})=\boldsymbol{\Gamma} \mathbf{J}\left(\mathbf{r}_{s}\right),
$$

where $\boldsymbol{\Gamma}$ is the operator matrix

$$
\boldsymbol{\Gamma}=\left[\begin{array}{ll}
-\hat{n} \times \nabla \times\left[G\left(\mathbf{r} ; \mathbf{r}_{s}\right) *\right. & \hat{n} \times j \omega \mu\left(1+\nabla^{2} / k^{2}\right)\left[G\left(\mathbf{r} ; \mathbf{r}_{s}\right) *\right. \\
\hat{n} \times j \omega \epsilon\left(1+\nabla^{2} / k^{2}\right)\left[G\left(\mathbf{r} ; \mathbf{r}_{s}\right) *\right. & \hat{n} \times \nabla \times\left[G\left(\mathbf{r} ; \mathbf{r}_{s}\right) *\right.
\end{array}\right]
$$

establish the conventions used in this paper and discuss our formulation of the coupled integral equations for twodimensional diffraction. We will describe in detail three adaptations that we made to the standard integral method. They are the following: our choice of the basis function expansion of the surface currents, the calculation of the incident fields, and the calculation of the diffracted far field by using the calculated surface currents. An important part of any complicated numerical calculation is testing the numerical results. Therefore we also include a detailed discussion of the various tests that we used, including a new test that takes advantage of our choice of
In the above operator, $G\left(\mathbf{r} ; \mathbf{r}^{\prime}\right)$ is the free-space Green's function

$$
G\left(\mathbf{r} ; \mathbf{r}^{\prime}\right)=\frac{\exp \left(j k\left|\mathbf{r}-\mathbf{r}^{\prime}\right|\right)}{4 \pi\left|\mathbf{r}-\mathbf{r}^{\prime}\right|}
$$

where $k=\omega \sqrt{\mu \epsilon}$ and the symbol $*$ in the operator represents the convolution

$$
G\left(\mathbf{r} ; \mathbf{r}_{s}\right) * \mathbf{J}\left(\mathbf{r}_{s}\right)=\oint \oint G\left(\mathbf{r} ; \mathbf{r}_{s}\right) \mathbf{J}\left(\mathbf{r}_{s}\right) \mathrm{d} \mathbf{r}_{s}
$$


By writing Eq. (2) for each medium and the incident field and applying boundary conditions at the surface, one can form two coupled integral equations for the tangential fields at the surface. In the operator notation, the result is

$$
\left[\Gamma^{(1)}-\Gamma^{(0)}-\mathbf{1}\right] \mathbf{J}=-\mathbf{J}^{(i)} .
$$

The superscript 1 or 0 for the operator $\Gamma$ indicates that the Green's function for medium 1 or 0 , respectively, is used. $\mathbf{J}^{(i)}$ are the surface currents resulting from the known incident fields. To solve these coupled integral equations numerically, a set of basis functions is chosen to express the surface currents, and the known and unknown quantities of the integral equations become vectors of coefficients. In addition, the operators in Eq. (6) become a matrix of coefficients, where each matrix element includes a numerical integration of a Green's function and a basis function. Inverting this matrix gives the solution vector of unknown surface current coefficients. To obtain the far-field solution, a final numerical integration is calculated by using the solution surface currents and a Green's function simplified for the far-field calculation.

For our analysis we simplify the integral equations to two dimensions. In this case the diffracting structures are independent of the third dimension ( $y$ axis), and they represent infinitely long grooves. Also, the incident fields are independent of the third dimension, and this restriction implies that the direction of propagation of the incident field has no $\hat{y}$ component. Thus our focused spot will be formed by a cylindrical lens, and the diffracted far field is calculated only for a one-dimensional angular spectrum, corresponding to a line in the Fourier plane of the objective lens. We justify the use of a twodimensional model with the observation that the pits embossed on optical disks are subwavelength only in the radial (cross-track) direction and that they are generally longer than a spot size in the azimuthal direction. Similarly, interconnect lines in semiconductor circuits are generally much longer than a spot size.

When the surface and all the fields are independent of a particular direction, say the $\hat{y}$ direction, then the integrals can be immediately performed along the $y$ axis because only the Green's function has any dependence on $y$. The Green's function becomes the two-dimensional Green's function

$$
G\left(\mathbf{r} ; \mathbf{r}_{s}\right)=\int_{-\infty}^{\infty} \frac{\exp \left(j k\left|\mathbf{r}-\mathbf{r}_{s}\right|\right)}{4 \pi\left|\mathbf{r}-\mathbf{r}_{s}\right|} \mathrm{d} y=\frac{j}{4} H_{0}^{(1)}\left(k\left|\mathbf{r}-\mathbf{r}_{s}\right|\right),
$$

which is the Hankel function of the first kind and order zero. The remaining integration required to calculate each matrix element is a contour integral in the $x-z$ plane. Since all the integrations are now contour integrations, the independent variable is better described as a scalar $s$, which is the length along the contour starting from the point on the contour where $x=0$ (see Fig. 2). The value of $s$ at any point along the contour is calculated in the standard manner: $s(x)=\int_{0}^{x}(\mathrm{~d} s / \mathrm{d} x)\left(x^{\prime}\right) \mathrm{d} x^{\prime}$. The unit vectors $\hat{\tau}$ and $\hat{n}$, which point tangentially and normally, respectively, to the contour, will ease the notation for the formulation in two dimensions. We have chosen the direction of $\hat{\tau}$ so that $\hat{\tau} \times \hat{n}=\hat{y}$. Another consequence of the independence in the $y$ direction is that the problem splits into two independent polarizations: $\mathbf{E}$ $=E \hat{y}(\mathrm{TE})$ and $\mathbf{H}=H \hat{y}(\mathrm{TM})$. These polarizations are independent because an incident field with one of these polarizations will scatter only to fields of the same polarization.

The operators (3) can be simplified for the twodimensional case. For the reduction of the integral equations, we will assume the TE polarization. The results for the TM polarization are in Appendix A. Knowing the specific directions of the surface currents, we can simplify the matrix of operators. Substituting these surface currents into the matrix elements, we obtain

$$
\begin{aligned}
\Gamma_{11} \mathbf{J}_{s, m}\left(\mathbf{r}_{s}\right)= & \hat{n}(s) \times \nabla \times \int_{-\infty}^{\infty}\left[G_{1}(R)-G_{0}(R)\right] \\
& \times J_{s, m}\left(s^{\prime}\right) \hat{\tau}\left(s^{\prime}\right) \mathrm{d} s^{\prime}, \\
\Gamma_{12} \mathbf{J}_{s, e}\left(\mathbf{r}_{s}\right)= & j \omega \mu \hat{n}(s) \times \int_{-\infty}^{\infty}\left[G_{1}(R)-G_{0}(R)\right] \\
& \times J_{s, e}\left(s^{\prime}\right) \hat{y} \mathrm{~d} s^{\prime}, \\
& \hat{n}(s) \times\left\{\int_{-\infty}^{\infty}\left[k_{1}^{2} G_{1}(R)-k_{0}{ }^{2} G_{0}(R)\right]\right. \\
\boldsymbol{\Gamma}_{21} \mathbf{J}_{s, m}\left(\mathbf{r}_{s}\right)= & j \omega \mu \\
& \times J_{s, m}\left(s^{\prime}\right) \hat{\tau}\left(s^{\prime}\right) \mathrm{d} s^{\prime}+\nabla^{2} \\
& \int_{-\infty}^{\infty}\left[G_{1}(R)-G_{0}(R)\right] \\
& \left.\times J_{s, m}\left(s^{\prime}\right) \hat{\tau}\left(s^{\prime}\right) \mathrm{d} s^{\prime}\right\} \\
& \times J_{s, e}\left(s^{\prime}\right) \hat{y} \mathrm{~d} s^{\prime}, \\
\boldsymbol{\Gamma}_{22} \mathbf{J}_{s, e}\left(\mathbf{r}_{s}\right)= & \hat{n}(s) \times \int_{-\infty}^{\infty}\left[G_{1}(R)-G_{0}(R)\right] \\
&
\end{aligned}
$$

where we have assumed that $\mu=\mu_{1}=\mu_{0}$ and used $R$ $=\left|\mathbf{r}_{s}-\mathbf{r}_{s^{\prime}}\right|$.

In order to form a matrix of coefficients, we expand the surface current on a set of basis functions. In Refs. 1 and 2 , the surface currents are assumed to be constant over small segments, making the basis functions rect functions $[\operatorname{rect}(x)=1$ when $|x|<1 / 2$, and 0 otherwise]. This approach works well when the incident field has a small angular spectrum, such as a plane wave. However, when the incident field is a tightly focused spot, then the number of grid points required to describe the incident field satisfactorily becomes very large, even when the diffract-

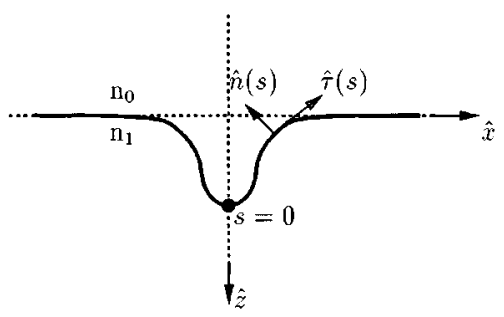

Fig. 2. Diffracting structure and coordinate systems for a twodimensional problem. 
ing surface is a flat plane. In the present case, the incident field is a focused spot resulting from a lens of finite aperture, and so the angular spectrum (spatial bandwidth) of the incident field is limited to the numerical aperture of the focusing lens. A good choice of basis functions might then be sinc functions $[\operatorname{sinc}(x)=(\sin x) / x$ ]. The Nyquist sampling theorem states that a function of finite bandwidth (angular spectrum) is identically equal to a sum of evenly spaced samples weighted by sinc functions if the sample rate is at least twice the highest frequency (plane-wave angle) of the function. That is, $f(x)$ $=\Sigma_{l} f(l \pi / W) \operatorname{sinc}(\pi W x-\pi l)$ if $W$ is greater than or equal to the bandwidth of $f(x)$. As a result, the incident field and the surface currents can be perfectly represented by a set of sinc basis functions if the diffracting surface is a flat plane. For diffracting surfaces that are not a flat plane, however, the incident field is no longer a simple Fourier transform of its angular spectrum, and a sampling rate greater than the Nyquist frequency is normally required. This higher sampling rate corresponds to allowing for evanescent waves in other numerical approaches, such as coupled-wave analysis. In general, the sample rate required for good results increases as the surface becomes less like a flat surface. The disadvantage of sinc basis functions is that the integral in each of the matrix elements now has infinite limits, whereas with rect basis functions, the integral is only over the region where the rect functions are nonzero. Compared with rect basis functions as used in Refs. 1 and 2, sinc basis functions are superior for flat and nearly flat surfaces. However, for deep and narrow grooves in the diffracting surface, the better basis function is not clear.

The expansion of the surface currents is of the form

$$
\begin{gathered}
J_{s, m}(s)=\sum_{l=-N}^{N} a_{l} \operatorname{sinc}\left(k_{0} W s-\pi l\right), \\
J_{s, e}(s)=\sum_{l=-N}^{N} b_{l} \operatorname{sinc}\left(k_{0} W s-\pi l\right) .
\end{gathered}
$$

The unaliased bandwidth of the surface currents is determined by $W$. The coefficients $a_{l}$ and $b_{l}$ are samples of the surface currents at the grid points $s=\pi l / k_{0} W$. We have truncated the expansion to $2 N+1$ terms. Substituting the basis functions into the matrix elements and placing the coefficients in the vector of unknowns, we obtain

$$
\begin{aligned}
\Gamma_{11_{l}}= & -\hat{n}(s) \times \nabla \times \int_{-\infty}^{\infty}\left[G_{1}(R)-G_{0}(R)\right] \\
& \times \operatorname{sinc}\left(k_{0} W s^{\prime}-\pi l\right) \hat{\tau}\left(s^{\prime}\right) \mathrm{d} s^{\prime} \\
\Gamma_{12_{l}}= & j \omega \mu \hat{\tau}(s) \int_{-\infty}^{\infty}\left[G_{1}(R)-G_{0}(R)\right] \\
& \times \operatorname{sinc}\left(k_{0} W s^{\prime}-\pi l\right) \mathrm{d} s^{\prime}
\end{aligned}
$$

$$
\begin{aligned}
\Gamma_{21_{l}}= & \frac{\hat{n}(s)}{j \omega \mu} \times\left\{\int_{-\infty}^{\infty}\left[k_{1}{ }^{2} G_{1}(R)-k_{0}^{2} G_{0}(R)\right]\right. \\
& \times \operatorname{sinc}\left(k_{0} W s^{\prime}-\pi l\right) \mathrm{d} s^{\prime} \\
& +\nabla^{2} \cdot \int_{-\infty}^{\infty}\left[G_{1}(R)-G_{0}(R)\right] \\
& \left.\times \operatorname{sinc}\left(k_{0} W s^{\prime}-\pi l\right) \mathrm{d} s^{\prime}\right\} \hat{\tau}\left(s^{\prime}\right), \\
\Gamma_{22_{l}}= & \hat{y}[\hat{n}(s) \cdot \nabla] \int_{-\infty}^{\infty}\left[G_{1}(R)-G_{0}(R)\right] \\
& \times \operatorname{sinc}\left(k_{0} W s^{\prime}-\pi l\right) \mathrm{d} s^{\prime},
\end{aligned}
$$

and the vector of unknowns is $\mathbf{J}=\left[\begin{array}{ll}a_{l} & b_{l}\end{array}\right]^{\prime}$. The integral equations can now be discretized to form the matrix equation. Since the choice of sinc functions as the basis for the surface currents guarantees that the resulting surface currents will be band limited, the integral equations can be discretized without any further loss of accuracy by simply sampling at the grid points.

The calculation of the matrix elements [Eqs. (10)] contains both derivative operators and integration. Reversing the order of differentiation and integration would be convenient, but this technique cannot be used if the integration is across an integrable singularity. The integrands in Eqs. (10) have an integrable singularity for the matrix elements along the diagonal. These matrix elements are for the cases in which the field grid point (Green's function) and the source grid point (sinc function) are the same. To integrate these integrands numerically, we used the technique of subtracting the singularity from the integrand and integrating it separately in closed form. ${ }^{21,22}$ This technique is possible because the Hankel function has a logarithmic singularity that can be integrated in closed form.

\section{B. Incident Field}

The right-hand side of the general matrix equation (6) is the tangential component of the incident field calculated at the grid points $s_{m}$. Many different models for the incident fields can be used. However, restrictions must be placed on the incident field if the numerical problem is to remain practical. As we mentioned in the formulation in the previous subsection, one source of error is the truncation of the region covered by the grid points. The truncation is reasonable only if the power flow across the surface beyond the covered region is negligible. Therefore the size of the matrix equation can be kept small only if the power flow of the incident field is concentrated over a relatively small region. For example, plane waves do not meet this criterion, but focused spots do.

Figure 1 shows a lens focusing light onto the diffracting surface. The fields focused by the lens can be expressed as an integral of plane waves. ${ }^{23-27}$ If $B(u)$ represents the amplitude and the phase of the TE electric field at the entrance pupil of the lens, where $u$ is the transverse coordinate in this plane, then the incident field behind the lens is 


$$
\mathbf{E}^{(i)}(x, z)=\hat{y} \int B(u) \exp \left[j k_{0}\left(u x+\sqrt{1-u^{2}} z\right)\right] \mathrm{d} u .
$$

The incident magnetic field follows from Maxwell's equations:

$$
\begin{aligned}
\mathbf{H}_{x}^{(i)}(x, z)= & \int B(u) \sqrt{1-u^{2}} \\
& \times \exp \left[j k_{0}\left(u x+\sqrt{1-u^{2}} z\right)\right] \mathrm{d} u, \\
H_{z}^{(i)}(x, z)= & \int B(u) u \\
& \times \exp \left[j k_{0}\left(u x+\sqrt{1-u^{2}} z\right)\right] \mathrm{d} u .
\end{aligned}
$$

Since the focal point is near the origin, $x$ and $z$ are always small enough so that the integrals for the incident fields are easily calculated by direct numerical integration. In most of our simulations, we used

$$
B(u)= \begin{cases}1, & |u| \leqslant \mathrm{NA} \\ 0, & |u|>\mathrm{NA}\end{cases}
$$

where NA corresponds to the numerical aperture of the lens. Another, perhaps more accurate, choice might be

$$
B(u)=\left\{\begin{array}{cl}
1 / \sqrt{1-u^{2}}, & |u| \leqslant \mathrm{NA} \\
0, & |u|>\mathrm{NA}^{\prime}
\end{array}\right.
$$

as is discussed by Mansuripur. ${ }^{23}$ The incident surface currents for the right-hand side of the matrix equation are then

$$
\begin{aligned}
\mathbf{J}_{s, m}^{(i)}(s)= & \hat{n} \times \mathbf{E}^{(i)}=\hat{\tau}(s) E_{y}^{(i)}\left(x_{s}, z_{s}\right), \\
\mathbf{J}_{s, e}^{(i)}(s)= & -\hat{n} \times \mathbf{H}^{(i)}=\hat{y}\left[n_{x}(s) H_{z}^{(i)}\left(x_{s}, z_{s}\right)\right. \\
& \left.-n_{z}(s) H_{x}^{(i)}\left(x_{s}, z_{s}\right)\right] .
\end{aligned}
$$

The incident fields for the TM case are completely analogous: $H_{y}(x, z)$ is calculated from the front focal plane distribution, and the electric-field components are derived from the curl of $\mathbf{H}$.

\section{Far Field}

Once the matrix elements and the incident-field vector are calculated, the matrix equation is solved by using a standard numerical method such as lower triangularupper triangular decomposition. The solution vector contains the coefficients $a_{l}$ and $b_{l}$, which can be substituted into Eq. (9) to find the surface currents anywhere on the contour. Unfortunately, the tangential fields at the contour cannot be directly measured. Useful results of this diffraction calculation would be the amplitude and the phase in the far field, since these quantities can be experimentally measured. To calculate the far field, we make a far-field approximation of the integral equations to calculate the scattered electric field (magnetic field in the TM case). For this calculation we use a far-field approximation for the Hankel function, and we simplify the integrand by ignoring terms that have a dependence on $\mathbf{r}$ stronger than $1 / \sqrt{|\mathbf{r}|}$. The electric far field can then be calculated by a simple numerical integration.

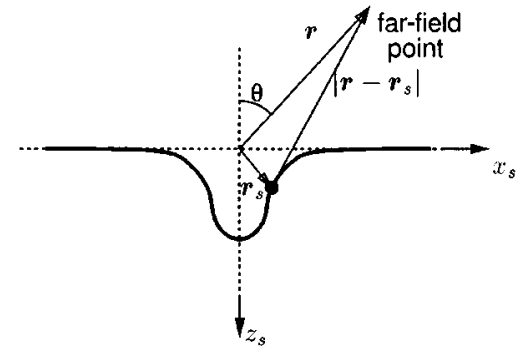

Fig. 3. Coordinate system for the far-field calculation.

If we ignore a constant factor of $\exp \left(j k_{0} r\right) / \sqrt{r}$, the electric far field at angle $\theta$ (see Fig. 3), or at the equivalent point in the Fourier plane of an objective lens, is equal to

$$
\begin{aligned}
-\mathbf{E}(\theta)= & \hat{y} \\
& \times \int_{-\infty}^{\infty}\left\{\left[n_{x}\left(s^{\prime}\right) \sin \theta-n_{z}\left(s^{\prime}\right) \cos \theta\right]\right. \\
& \left.\times J_{s, m}\left(s^{\prime}\right)+J_{s, e}\left(s^{\prime}\right)\right\} \\
& \times \exp \left\{-j k_{0}\left[x\left(s^{\prime}\right) \sin \theta-z\left(s^{\prime}\right) \cos \theta\right]\right\} \mathrm{d} s^{\prime} .
\end{aligned}
$$

The integration in the above formula is easily performed numerically because all the grid points and the surface currents have already been tabulated. Values for $\theta$ can be chosen arbitrarily, although values that lie within the numerical aperture of the objective lens used to collect the scattered light are generally used.

\section{Testing the Numerical Results}

Before we can start using a computer program to predict diffraction behavior, we must have some confidence that the program is both numerically stable and an accurate representation of the mathematical formulation. The literature suggests several methods for testing the numerical results. 10,20 These methods include comparing numerical results with analytic solutions for the small class of known solutions, checking that the numerical solution satisfies the appropriate power conservation laws, testing reciprocity relations with the numerical method, testing the numerical results with the extinction cross-section theorem, and checking the convergence of the numerical results for decreasing grid spacing and increasing matrix rank.

We found that different checks were useful at different stages of forming the numerical computer program. For the problem of a planar interface, a problem for which analytic solutions are known, only the matrix elements of $\boldsymbol{\Gamma}_{12}$ and $\boldsymbol{\Gamma}_{21}$ are nonzero. We took advantage of this fact by using the planar interface problem to scrutinize the calculation of the matrix elements $\boldsymbol{\Gamma}_{21}$, the most complicated calculations and therefore the most likely to contain errors. Power conservation relations, discussed in more detail in the Subsection 2.D.1, proved useful when putting together the various components of the overall calculation. If, for example, a sign error were present in one of the incident fields, then power conservation would not follow. These two tests ensure that the computer program 
is an error-free representation of the formulated equations. However, they do not, in general, test the numerical stability and accuracy of the computer program. The best method to evaluate the numerical stability of the computer program is simply to run the program repeatedly for the same problem with an increasing number of grid points. If the numerical solution converges in a reasonable manner, then the numerical program is stable, at least for the particular problem that was computed. The numerical accuracy of the solution can be estimated from the convergence data. The last check that we will discuss is a new check specifically for the parameter $W$, the scale of the sinc functions and thus the inverse of the grid spacing. This method uses the fast Fourier transform (FFT) of the surface currents to check the validity of the assumption that they are band limited to $W$.

\section{Power Flow}

Power conservation dictates that the time-averaged power flow transmitted across the contour boundary plus the power reflected from the boundary is equal to the power in the incident field. Unlike formulations that use a plane wave as the incident field, our treatment can use only incident fields with finite power. In this subsection we will first show that the power flow across a boundary can be calculated with the surface currents. Applying this formulation and the orthogonality of the sinc functions, we will derive the formulas for calculating the transmitted, reflected, and incident power from the surface current coefficients.

The time-averaged electromagnetic power flow across a surface is found by integrating the normal component of the real part of the Poynting vector ${ }^{28}$ :

$$
\langle P\rangle=\int_{-\infty}^{\infty} \operatorname{Re}\left(\mathbf{E} \times \mathbf{H}^{*}\right) \cdot \hat{n} \mathrm{~d} s .
$$

Having formulated the problem in two dimensions, we are ignoring the integration in the $\hat{y}$ direction. However, the normal component of the Poynting vector can be represented by using surface currents. From the definition of the surface currents, it follows that

$$
\mathbf{J}_{s, m} \times \mathbf{J}_{s, e}^{*}=(-\hat{n})\left[\hat{n} \cdot\left(\mathbf{E} \times \mathbf{H}^{*}\right)\right] .
$$

In our two-dimensional treatment, we already know the directions of the surface current vectors. For the TE case, we have

$$
J_{s, m} \hat{\tau} \times J_{s, e}^{*} \hat{y}=-J_{s, m} J_{s, e}^{*} \hat{n},
$$

and the time-averaged power flow across the surface is

$$
\langle P\rangle=\int_{-\infty}^{\infty} \operatorname{Re}\left[J_{s, m}(s) J_{s, e}^{*}(s)\right] \mathrm{d} s .
$$

Note that we have changed the sign of the integrand so that the power flow is in the direction opposite to the direction of $\hat{n}$. Substituting the basis function expansions of the surface currents [Eqs. 9] into the expression for the average power [Eq. (20)], we obtain the power flow in terms of the surface current coefficients:

$$
\langle P\rangle=\frac{\pi}{2 W} \sum_{l} \operatorname{Re}\left(a_{l} b_{l}^{*}\right) .
$$

This last formula results from the orthogonality relation

$$
\begin{aligned}
\int_{-\infty}^{\infty} \operatorname{sinc}\left(k_{0} W s^{\prime}-\pi l\right) \operatorname{sinc}\left(k_{0} W s^{\prime}-\pi l^{\prime}\right) \mathrm{d} s^{\prime} & \\
& =\frac{\pi}{2 k_{0} W} \delta_{l l^{\prime}}
\end{aligned}
$$

where $l$ and $l^{\prime}$ are integers and $\delta_{l l^{\prime}}=1 \Leftrightarrow l=l^{\prime}$.

The power calculated in Eq. (21) gives the total power flow across the boundary, which is the transmitted power. To test the power conservation relation

$$
\langle P\rangle=\left\langle P^{(i)}\right\rangle-\left\langle P^{(r)}\right\rangle
$$

we also need to calculate the incident power $\left\langle P^{(i)}\right\rangle$ and the reflected power $\left\langle P^{(r)}\right\rangle$. The incident power is simply the power flow across the boundary for the case in which there is no optical contrast between the two regions. In that case $a_{l}=a_{l}^{(i)}$ and $b_{l}=b_{l}^{(i)}$. Substitution of these coefficients, already calculated as the right-hand side of the matrix equation, into Eq. (21) gives $\left\langle P^{(i)}\right\rangle$. To calculate the reflected power, we must first separate the tangential fields into incident and scattered fields:

$$
\begin{gathered}
\mathbf{J}_{s, m}(s)=\mathbf{J}_{s, m}^{(i)}(s)+\mathbf{J}_{s, m}^{(r)}(s), \\
\mathbf{J}_{s, e}(s)=\mathbf{J}_{s, e}^{(i)}(s)+\mathbf{J}_{s, e}^{(r)}(s) .
\end{gathered}
$$

If the surface currents for the reflected fields are expanded on the same sinc basis set, the orthogonality of the basis functions gives

$$
a_{l}^{(r)}=a_{l}-a_{l}^{(i)}, \quad b_{l}^{(r)}=b_{l}-b_{l}^{(i)} .
$$

The reflected power can now be calculated by using the coefficients $a_{l}^{(r)}$ and $b_{l}^{(r)}$ in Eq. (21).

\section{Convergence}

Convergence of the numerical results means that if a calculation is repeated several times with a smaller grid spacing each time, then the numerical results will become more accurate with each repetition. In our case we must test convergence for both decreasing grid spacing (increasing $W$ ) and increasing number of grid points with a fixed grid spacing. The latter test is required because we do not have a periodic structure, and we truncated the integral to finite limits. Convergence testing is important and must always be performed before trustworthy results can be obtained. In every numerical calculation, there is a tradeoff between accuracy and computation time. By considering the convergence properties, one can choose a grid spacing that gives satisfactory accuracy for a reasonable computation time. Naturally, the convergence estimation itself will require a long computation time, since the test must necessarily extend to grid spacing smaller than required. Also, the test must be carried to grid spacings small enough to determine if the results are oscillating or converging uniformly. Finally, the numerical results for the surface currents and the far field will converge at different rates for different contour points or farfield angles, depending on the particular contour. 


\section{Checking the Sample Rate with the Fast Fourier Transform}

The choice of sinc functions for the basis functions of the surface current allows for a new type of convergence check. As we stated above, expanding a function on a basis set of sinc functions makes the implicit assumption that the function is strictly band limited. Also, the coefficients $a_{l}$ and $b_{l}$ are samples of the surface currents at the points $s=\pi l / k_{0} W$. If the surface currents are, in fact, band limited, then their Fourier transforms, which can be calculated since we know the functions exactly everywhere, should show a cutoff frequency. If we calculate the (FFT's) of the surface current coefficients, the highest-frequency bin will correspond to the cutoff frequency of the band-limited functions. Therefore, if the FFT's of the surface current coefficients have significant amplitudes in the high-frequency bins, then we can reasonably assume that there is aliasing and that the grid spacing needs to be shortened (the bandwidth $W$ needs to be increased).

\section{EXPERIMENTAL VERIFICATION}

Before we can confidently use our numerical method to predict the diffraction behavior for various structures and incident fields, we would like to compare some calculations with actual diffraction measurements. Figure 4 diagrams the laboratory setup. The iris between 11 and 12 is imaged onto the entrance pupil of the objective lens, reducing the numerical aperture of the incident field. The lateral extent of the focused incident spot on the sample is approximately $1.1 \mu \mathrm{m}$ when the numerical aperture is set to 0.6. The full numerical aperture of the objective lens is used to capture the reflected field. The back focal plane, or Fourier plane, of the objective is imaged by 14 and 15 onto the CCD camera, where an intensity profile is measured. The reference mirror is used to focus the illumination spot, and it is blocked when diffraction measurements are recorded.

We measure the intensity in the Fourier plane for a three-dimensional spot, but our numerical calculation is only for two dimensions. If the test pattern is independent of the $\hat{y}$ direction, then we can argue that no plane wave propagating in a direction with a nonzero $\hat{y}$ component can be diffracted in a direction with a zero $\hat{y}$ component. Therefore our calculations are in reality for the $x$ axis of the Fourier plane, which represents the spectrum of plane waves with no $\hat{y}$ component. For our experimental results, we use a cross section of the measured Fourier plane along the $x$ axis.

The test sample consists of sinusoidal gratings in aluminum. The gratings were fabricated by the Rochester Photonics Corporation by using a rastered laser spot to expose a photoresist. After the photoresist was developed to form the grating, a coating of approximately 50 $\mathrm{nm}$ of aluminum was evaporated onto the grating. Finally, the actual depth and profile of the grating were measured with an atomic force microscope at the Jet Propulsion Laboratory. The surface measurement showed that the grating was sinusoidal with a peak-to-valley height of $0.185 \mu \mathrm{m}$. The grating period was $1.0 \mu \mathrm{m}$.

For this grating the Wood's anomaly should be quite evident. We can estimate the angular location where we expect to see strong absorption by calculating which plane-wave angle is coupled into the plane wave traveling parallel to the surface by the grating. Using the Floquet condition $\sin \theta_{r}=\sin \theta_{i}+i \lambda / d$, where $\theta_{r}$ is the reflection angle, $\theta_{i}$ is the incidence angle, and $i$ is an integer, and setting the reflection angle to $\sin \theta_{r}=1$, we expect the anomalous absorption at the incidence angles

\begin{tabular}{cc}
$i$ & $\sin \theta_{i}=1-i \lambda / d$ \\
\hline+1 & 0.37 \\
+2 & -0.27
\end{tabular}

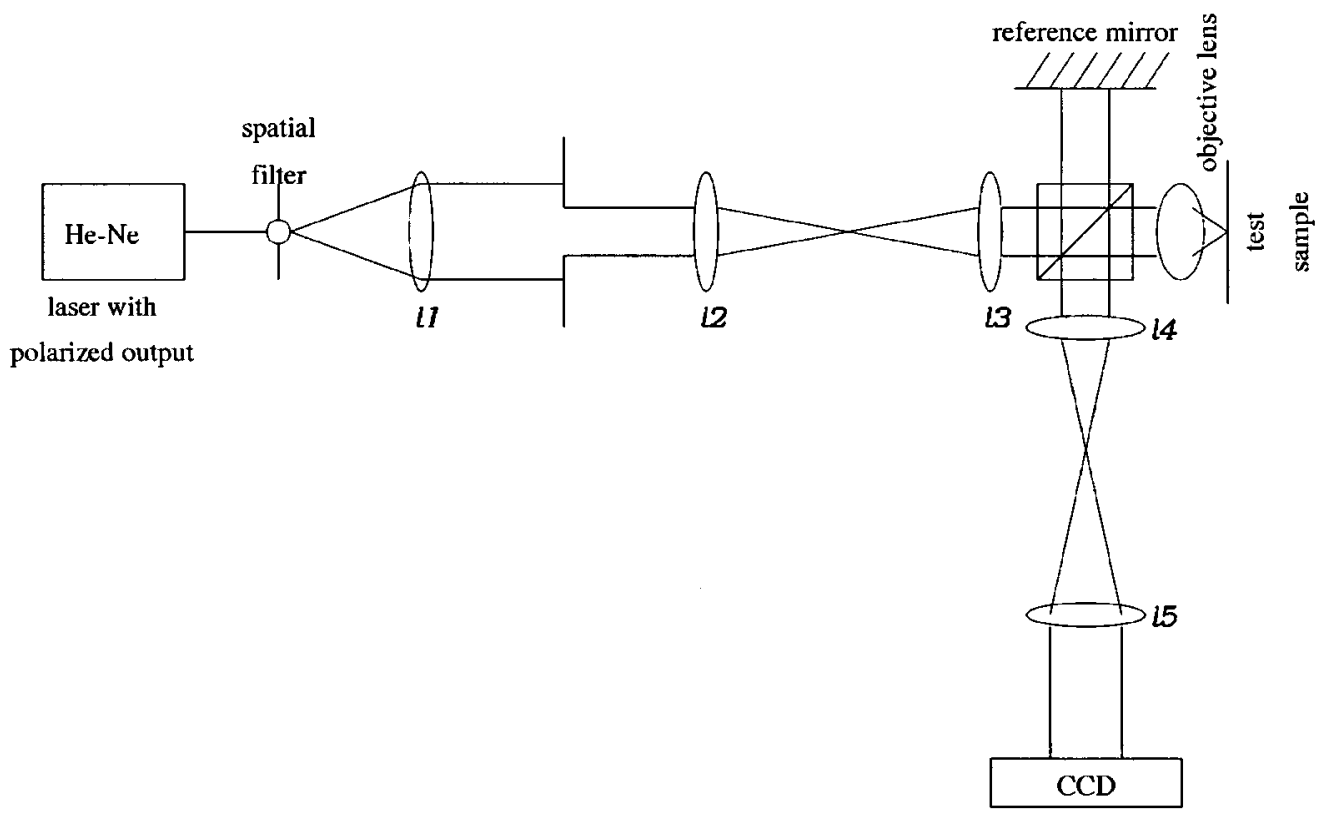

Fig. 4. Setup for measurement of diffracted far field for focused incident fields. 


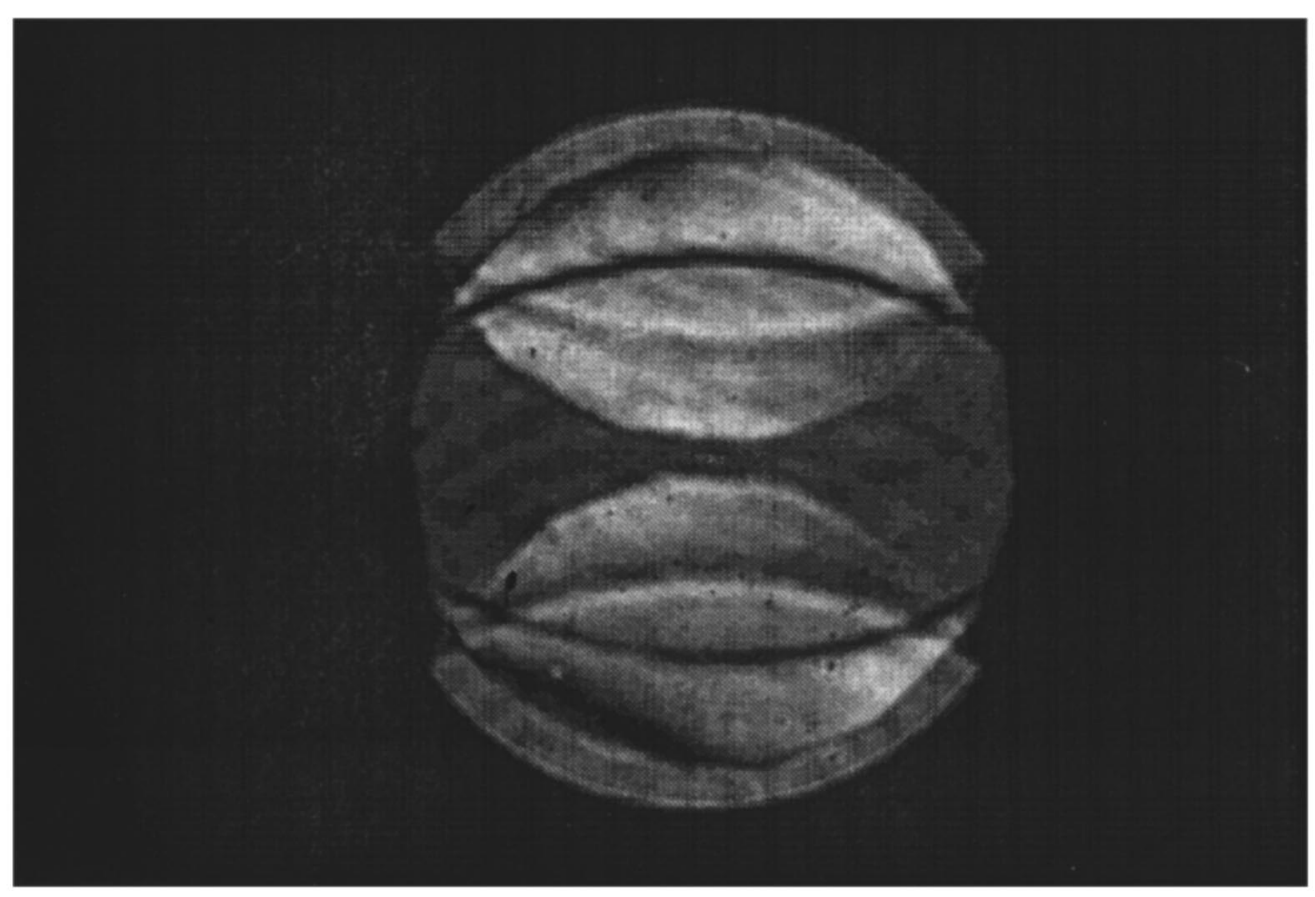

Fig. 5. Reflected Fourier plane for aluminum grating with TM incidence, clearly showing the Wood's anomalies.

for this aluminum grating with $\lambda=0.6328 \mu \mathrm{m}$. Measurements of the Wood's anomalies presented in Ref. 10 show that the actual anomalous angles are slightly different from those estimated by the above method.

In our measurements we use an objective lens to form a focused spot on the grating. The focused spot is composed of a superposition of all the plane waves with angles that are less than the numerical aperture of the focusing objective lens. ${ }^{25,27}$ Also, the Fourier plane (back focal plane) of the objective is a map of the far field of the focal plane, so that the field at coordinate $x_{f}$ in the Fourier plane equals the far field at angle $\theta$ with the mapping $\sin \theta \approx x_{f} / f$, where $f$ is the focal length of the objective lens. Consequently, we expect to measure notches in the reflected intensity in the Fourier plane corresponding to the anomalous far-field angles, even while the grating is being illuminated with all the superimposed plane waves simultaneously.

Figure 5 shows the CCD image of the Fourier plane for a focused TM spot on the aluminum grating. The image is oriented with the $x_{f}$ axis in the vertical direction, and it contains several interesting features. The numerical aperture for the incident spot was approximately 0.6 , which is equal to the maximum $\sin \theta$ of the incident plane waves. The +1 and -1 diffracted orders are shifted $\sin \theta=\lambda / d=0.6328 / 1.0=0.6328$, thus forming the two partial circles that do not quite meet in the center. The faint complete circle that fills in the background is the zero-order reflected spot. Small bands at the top and the bottom of the zero-order spot are diffracted light captured because the numerical aperture for the collection of the reflected light was approximately 0.7 , which is larger than the numerical aperture for the incident light. Finally, the two dark arcs within each diffracted order result from the absorption of the Wood's anomalies. The darker band is for the +1 angle in the above table, and the other is for the +2 angle.

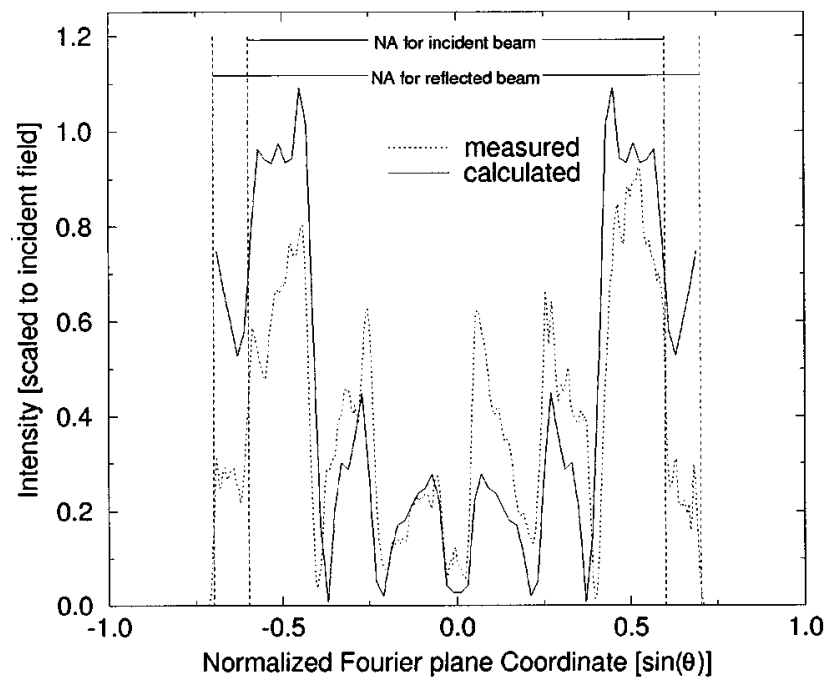

Fig. 6. Measurement versus calculation for the sinusoidal aluminum grating with the Wood's anomalies evident as minima in the angular spectrum of the reflected field. The aperture limitations to the incident and reflected fields are indicated by dashed vertical lines.

The numerical simulation for this problem was calculated for a sinusoidal surface in a medium with index of refraction $1.5+j 7.3{ }^{29}$ With the imaginary part of the index of refraction so large, the optical field is only approximately $2.7 \%$ of the incident amplitude at a depth of $50 \mathrm{~nm}$. Therefore approximating the thin film of aluminum by filling the whole volume with aluminum is reasonable. Figure 6 compares the numerical result with a vertical cross section taken from the image. Agreement is quite good at the smaller angles, and the nulls in the reflected power that are due to the Wood's anomalies are quite evident and occur very close to the predicted angles. The small differences in the location of the minima be- 
tween the numerical result and the measured cross section probably result from inaccuracy in scaling the abscissa of the measured values taken from the CCD image. The abscissa of the measured data was scaled so that the edge of the measured data corresponds to the specified numerical aperture of the objective lens. Inaccurate knowledge of the focal length and the numerical aperture of the objective lens contributes to the scaling error. In addition, the aperture of the incident field was set with a precision not better than $10 \%$. The vertical scale for each plot was derived by matching the measured and calculated reflections for a planar aluminum interface. We are pleased with the correspondence between the calculated and measured data.

\section{ANALYSIS OF THE DIGITAL VIDEO DISK FORMAT}

The improvement in track density from CD-audio to DVD is quite significant. Not only is the objective numerical aperture increased and the wavelength decreased to produce a smaller spot width, but the track pitch is significantly less than the spot width, and the pit width becomes less than half the wavelength. For the CD-audio format, the reflected signal caused by diffraction of the incident light with the track is adequately described by scalar explanations, and the difference between the TE and TM reflected fields is small. ${ }^{1,2}$ However, for the DVD format, the two polarizations show significant differences in the diffracted far field because of the reductions of the track pitch and the pit width with respect to the wavelength. This difference is best shown by the apparent pit depth, or phase depth. The phase depth is defined as $z_{p}=\lambda \phi / 2 \pi$, where $\phi$ is the phase retardation of the electric field at the bottom of the pit. In typical scalar diffraction approximations, the phase depth is equal to the pit depth. However, when the pit width is small, the phase depth can be significantly different from the actual depth. As is shown in Fig. 7, the phase of the TM field in a DVD pit is still an accurate representation of the actual pit depth, but the TE field does not seem to be as strongly affected. The central far-field intensity has the appropriate variation corresponding to the pit phase depth. This difference in the far-field intensity variation for the different polarizations stimulates an interesting idea for the doubling of the information density. Rather than each pit having two possible depths, zero or one-quarter wave as in the current DVD format, each pit can have four possible depths, and the depth can be distinguished by the relative far-field response of the TE and TM polarizations. The dashed vertical lines in the far-field plot of Fig. 7 indicate pit depths where four states are distinguishable. By placing a polarizing beam splitter in the return path and duplicating the standard detector array in each leg, we can measure the reflection of each polarization independently, doubling the information density of each pit.

Why should the TE polarization behave so differently for the CD-audio and DVD formats? The biggest change between the two formats, from the point of view of calculating diffraction, seems to be the pit width in relation to the wavelength. For both formats the pit width is approximately one third ( $\lambda / \mathrm{NA})$, where $\lambda$ is the wavelength

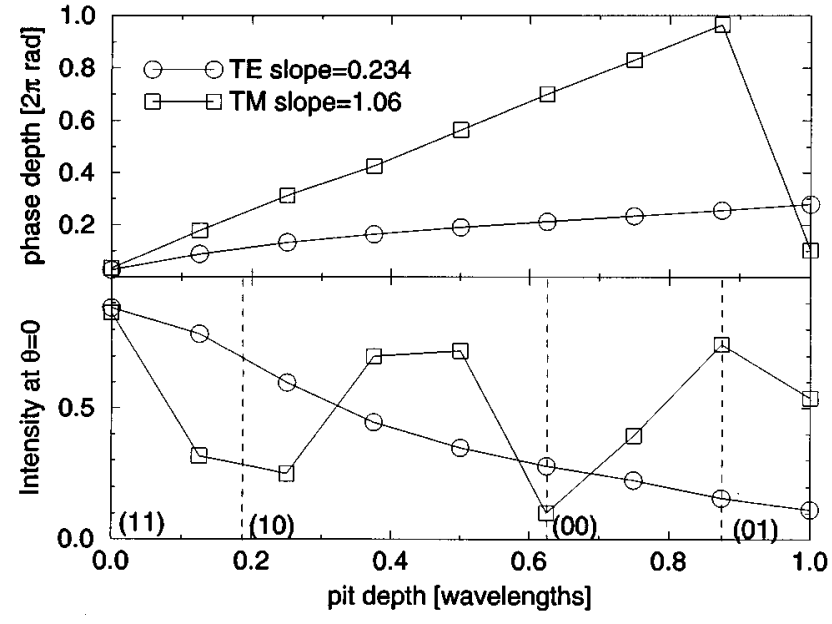

Fig. 7. Calculated pit phase depth and far-field intensity for the DVD format. The dashed vertical lines indicate which pit depths can be used to represent four logical states.

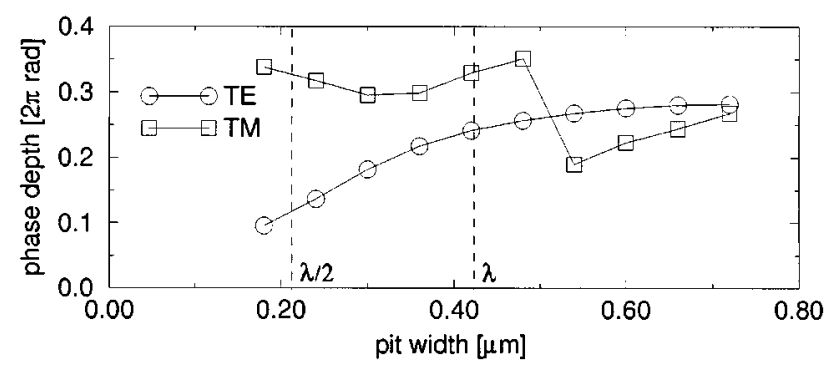

Fig. 8. Pit phase depth for different pit widths with the pit depth equal to a quarter wave. In the region where the pit width is between $\lambda / 2$ ( $\lambda$ is adjusted for the index of refraction of the incident medium) and $\lambda$, the phase depth for TE illumination varies almost a quarter wave, but it remains fairly constant for TM illumination.

and NA is the numerical aperture of the focusing objective. However, the numerical aperture increases by $1 / 3$ for the DVD format, thereby reducing the pit width in relation to the wavelength. To study the effect of changing the pit width, we calculated the pit phase depth for the DVD format as a function of pit width (Fig. 8). In the region where the pit width varies between $\lambda / 2$ and $\lambda$ (here $\lambda$ is the wavelength in the polycarbonate medium), the phase depth of the TM wave remains fairly constant, while the phase depth of the TE wave increases close to the quarter-wave depth of the pit. Therefore the pit width appears to be crucial to the penetration of the TE wave into the pit.

We can explain the difference between the TE and TM dependences on the pit width by considering the case of a plane, parallel waveguide with perfectly conducting walls. The fields in this waveguide consist of discrete modes, as restricted by the boundary conditions of the tangential electric field at the walls. The TE polarization for the pit corresponds to the electric field parallel to the walls of the waveguide, and the solutions for the electric field inside the waveguide are

$E_{y}=\sin (p \pi x / d) \exp \left(j k_{z} z\right), \quad k_{z}=\left[k^{2}-(p \pi / d)^{2}\right]^{1 / 2}$, 
where $d$ is the width of the waveguide and $p$ is an integer. When the magnetic field is parallel to the walls, the solutions are

$$
H_{y}=\cos (p \pi x / d) \exp \left(j k_{z} z\right), \quad k_{z}=\left[k^{2}-(p \pi / d)^{2}\right]^{1 / 2} .
$$

Each mode, corresponding to an integer value of $p$, has a cutoff width $d_{c}^{p}=p \pi / k$. For widths smaller than $d_{c}^{p}, k_{z}$ is imaginary, and the field amplitude weakens exponentially in $z$. For the mode $p=0$, the TE solution is $E_{y}$ $=0$; there can be no TE fields. However, there is a TM solution for $p=0: H_{y}=\exp (j k z)$. The cutoff width for the $p=1$ mode is $d_{c}^{1}=\lambda / 2$, and there are both TE and TM propagating solutions for this mode when $d>d_{c}^{1}$. Therefore, when $d<\lambda / 2$, only evanescent TE waves can exist, while the TM zero mode can still propagate. Here lies the essential difference between the TE fields and the TM fields in our pit width calculations described above. When the pit width falls below half a wavelength, the TE fields can no longer penetrate the pit, while the TM fields can still propagate down the pit and back. Our basic principle is that by making the pit width small, we can have pits that the TM field sees, and that the TE field does not. Thus, as diffracting structures become small, a new measurement technique, completely independent of the classical imaging resolution limits, can be used that is very sensitive to very small changes in the structure's dimensions.

\section{CONCLUSION}

We have developed a numerical tool for the calculation of optical diffraction for cases in which the incident field is a focused spot and the diffracting structure is a single structure or an aperiodic surface. Our approach uses the integral formulation to solve Maxwell's equations, and one important difference between our results and previously published results using the integral technique is our choice of basis function. Since the particular diffraction problems that we are addressing are for tightly focused incident spots, the surface currents are better described by a set of sinc functions, rather than rect functions, which are more typical in previous work. Ramifications of using sinc functions as the basis function include a band-limited description of the surface currents, ease of calculating power flow, and increased complexity for the numerical integrals when calculating the matrix coefficients.

To verify the utility of the numerical diffraction program, we compared numerical results with experimental measurements of the far-field intensity for a focused spot incident on an aluminum grating. This specific example was chosen because of the presence of the Wood's anomaly and because other numerical diffraction methods, such as rigorous coupled-wave analysis, have difficulty converging for this problem, as has been previously reported. The integral approach converged quickly for this problem, and the comparison between prediction and measurement was favorable.

Finally, we used the new integral method to predict the diffraction behavior of the proposed DVD format for the next generation of optical disks. Our analysis shows that the reflected signal for this format has a strong dependence on the polarization of the incident light.

\section{APPENDIX A: INTEGRAL EQUATIONS FOR THE TM CASE}

For the TM case, the directions of $\mathrm{J}_{s, e}$ and $\mathrm{J}_{s, m}$ are reversed and mirrored with respect to their directions for the TE case, so that

$$
\mathbf{J}_{s, e}=J_{s, e} \hat{\tau}(s), \quad \mathbf{J}_{s, m}=-J_{s, m} \hat{y} .
$$

Because of this antisymmetry, the matrix elements $\Gamma_{11}$ and $\boldsymbol{\Gamma}_{22}$ for the TM case are exactly mirrored to those for the $\mathrm{TE}$ case in the manner

$$
\Gamma_{11_{l}}^{\mathrm{TM}}=\Gamma_{22_{l}}^{\mathrm{TE}}, \quad \boldsymbol{\Gamma}_{22_{l}}^{\mathrm{TM}}=\boldsymbol{\Gamma}_{11_{l}}^{\mathrm{TE}} .
$$

The other matrix elements are

$$
\begin{aligned}
\Gamma_{12}^{\mathrm{TM}} \mathbf{J}_{s, e}\left(\mathbf{r}_{s}\right)= & j \omega \mu \hat{n}(s) \times\left\{\int_{-\infty}^{\infty}\left[G_{1}(R)-G_{0}(R)\right]\right. \\
& \times J_{s, e}\left(s^{\prime}\right) \hat{\tau}\left(s^{\prime}\right) \mathrm{d} s^{\prime} \\
& +\nabla^{2} \cdot \int_{-\infty}^{\infty}\left[\frac{G_{1}(R)}{k_{1}{ }^{2}}-\frac{G_{0}(R)}{k_{0}{ }^{2}}\right] \\
& \left.\times J_{s, e}\left(s^{\prime}\right) \hat{\tau}\left(s^{\prime}\right) \mathrm{d} s^{\prime}\right\}, \\
\Gamma_{21}^{\mathrm{TM}} \mathbf{J}_{s, m}\left(\mathbf{r}_{s}\right)= & \frac{-\hat{n}(s)}{j \omega \mu} \times \int_{-\infty}^{\infty}\left[k_{1}{ }^{2} G_{1}(R)-k_{0}{ }^{2} G_{0}(R)\right] \\
& \times J_{s, m}\left(s^{\prime}\right) \hat{y} \mathrm{~d} s^{\prime} .
\end{aligned}
$$

\section{ACKNOWLEDGMENTS}

We thank Dean Faklis and the Rochester Photonics Corporation for providing the sample used in the measurements described in this paper and Dan Wilson of the Jet Propulsion Laboratory for measurements of the grating. The research was supported, in part, by the U.S. Army Research Office and by the National Science Foundation Center for Neuromorphic Systems Engineering at the California Institute of Technology.

*Present address, Sight Systems, Inc., 3541 Old Conejo Road, \#119, Newbury Park, California 91320.

\section{REFERENCES}

1. J. G. Dil and B. A. Jacobs, "Apparent size of reflecting polygonal obstacles of the order of one wavelength," J. Opt. Soc. Am. 69, 950-960 (1979).

2. G. Bouwhuis, J. Braat, A. Huijser, J. Pasman, G. van Rosmalen, and K. S. Immink, Principles of Optical Disc Systems (Hilger, Bristol, UK, 1985), Chap. 6.

3. D. Maystre, "Rigorous theory of light scattering from rough surfaces," J. Opt. (Paris) 15, 43-51 (1984).

4. A. K. Fung and M. F. Chen, "Numerical simulation of scattering from simple and composite random surfaces," J. Opt. Soc. Am. A 2, 2274-2284 (1985).

5. G. S. White and J. F. Marchiando, "Scattering from a $V$-shaped groove in the resonance domain," Appl. Opt. 22, 2308-2312 (1983).

6. E. R. Mendez and K. A. O'Donnell, "Observation of depolar- 
ization and backscattering enhancement in light scattering from Gaussian random surfaces," Opt. Commun. 61, 91-95 (1987).

7. The National Technology Roadmap for Semiconductors (Semiconductor Industry Association, San Jose, Calif., 1994).

8. C. J. Raymond, M. R. Murnane, S. L. Prins, S. S. H. Naqvi, J. R. McNeil, and J. W. Hosch, "Multi-parameter CD measurements using scatterometry," in Metrology Inspection and Process Control for Microlithography X, S. K. Jones, ed., Proc. SPIE 2725, 698-709 (1996).

9. C. J. Raymond, S. S. H. Naqvi, and J. R. McNeil, "Scatterometry for CD measurements of etched structures," in $M e$ trology Inspection and Process Control for Microlithography $X$, S. K. Jones, ed., Proc. SPIE 2725, 720-728 (1996).

10. R. Petit, Electromagnetic Theory of Gratings, Vol. 22 of Topics in Current Physics (Springer-Verlag, Berlin, 1980).

11. M. Neviere, R. Petit, and M. Cadilhac, "About the theory of optical grating coupler-waveguide systems," Opt. Commun. 8, 113-117 (1973).

12. J. P. Hugonin and R. Petit, "A numerical study of the problem of diffraction at a non-periodic obstacle," Opt. Commun. 20, 360-364 (1977).

13. A. Wirgin, "A new theoretical approach to scattering from a periodic interface," Opt. Commun. 27, 189-194 (1978).

14. K. Knop, "Rigorous diffraction theory for transmission phase gratings with deep rectangular grooves," J. Opt. Soc. Am. 68, 1206-1210 (1978).

15. F. G. Kaspar, "Diffraction by thick, periodically modified gratings with complex dielectric constant," J. Opt. Soc. Am. 63, 37-45 (1973).

16. M. G. Moharam and T. K. Gaylord, "Rigorous coupled-wave analysis of planar-grating diffraction,” J. Opt. Soc. Am. 71, 811-818 (1981).
17. M. G. Moharam and T. K. Gaylord, "Diffraction analysis of dielectric surface-relief gratings," J. Opt. Soc. Am. 72, 1385-1392 (1982).

18. T. K. Gaylord and M. G. Moharam, "Planar dielectric grating diffraction theories," Appl. Phys. B 28, 1-14 (1982).

19. W. B. Veldkamp, G. J. Swanson, S. A. Gaither, C.-L. Chen, and T. R. Osborne, "Binary optics: a diffraction analysis," Project Rep. ODT-20 (Massachusetts Institute of Technology Lincoln Laboratory, Lexington, Mass. 1989).

20. A. T. De Hoop, Modern Topics in Electromagnetics and Antennas, PPL Conf. Publ. 13 (Peter Peregrinus Ltd., Stevenage, UK, 1977), Chap. 6.

21. W. H. Press, B. P. Flannery, S. A. Teukolsky, and W. T. Vetterling, Numerical Recipes in C (Cambridge U. Press, Cambridge, 1988)

22. L. M. Delves and J. L. Mohamed, Computational Methods for Integral Equations (Cambridge U. Press, Cambridge, 1985).

23. M. Mansuripur, "Distribution of light at and near the focus of high-numerical-aperture objectives: erratum," J. Opt. Soc. Am. A 10, 382-383 (1993).

24. J. A. Stratton, Electromagnetic Theory (McGraw-Hill, New York, 1941), Chap. 6.

25. M. Born and E. Wolf, Principles of Optics, 6th ed. (Pergamon, New York, 1980), Chap. 8.

26. E. Wolf, "Electromagnetic diffraction in optical systems. 1. An integral representation of the image field," Proc. R. Soc. London, Ser. A 253, 349-357 (1959).

27. J. Goodman, Introduction to Fourier Optics (McGraw-Hill, San Francisco, 1968).

28. R. F. Harrington, Time-Harmonic Electromagnetic Fields (McGraw-Hill, New York, 1961).

29. E. D. Palik, ed., Handbook of Optical Constants of Solids (Academic, Orlando, Fla., 1985). 